\title{
Effects of Superposed Continuous Diode Laser on Welding Characteristics for Aluminum Alloy in Pulsed Nd:YAG Laser Welding
}

\author{
Kazuya MIURA ${ }^{* 1}$, Yasuhiro OKAMOTO ${ }^{* 1}$, Tomokazu SAKAGAWA ${ }^{* 2}$, \\ Yoshiyuki UNO $^{* 1}$ and Shinichi NAKASHIBA ${ }^{* 2}$ \\ ${ }^{*}$ Graduate School of Natural Science and Technology, Okayama University, 3-1-1 Tsushimanaka, \\ Kita-ku, Okayama 700-8530, Japan \\ E-mail:miura@ntmlab.mech.okayama-u.ac.jp \\ ${ }^{* 2}$ Advanced Laser Research Laboratory, Kataoka Corporation, 2-14-27 Shin-yokohama, \\ Kohoku-ku, Yokohama 222-0033, Japan
}

\begin{abstract}
The efficient welding of aluminum alloy has been strongly required for lightening the electronic products and automobiles, and a pulsed Nd:YAG laser is widely used for its application. However, the low absorption of a Nd:YAG laser makes it difficult to weld the aluminum alloy efficiently. Therefore, a pulsed Nd:YAG laser of $1064 \mathrm{~nm}$ in wavelength and a continuous diode laser of $808 \mathrm{~nm}$ in wavelength were spatially superposed, since the absorption rate of the continuous diode laser to the aluminum alloy is much higher than that of a pulsed Nd:YAG laser. The maximum temperature of specimen surface in the vicinity of laser irradiation area increased $50 \mathrm{~K}$ by the superposition of continuous diode laser and pulsed Nd:YAG laser, and the energy of Nd:YAG laser was absorbed efficiently. Moreover, the thermal analysis indicated that the temperature of specimen surface with the continuous diode laser and the pre-heating pulse could be kept approximately $200 \mathrm{~K}$ higher than that without these processes. The combination of pre-heating pulse and diode laser made it possible to increase the penetration depth even from the beginning of laser scanning.
\end{abstract}

DOI:10.2961/jlmn.2011.03.0010

Keywords: pulsed Nd:YAG laser, LD, high-efficient welding, aluminum alloy, thermal analysis

\section{Introduction}

The welding of aluminum alloy has been required for the purpose of lightening the personal digital device, the automobile, and so on. In general, a pulsed Nd:YAG laser has been mainly used for the fine micro-welding of aluminum alloy. However, the absorption rate of a $\mathrm{Nd}$ :YAG laser $(\lambda=1064 \mathrm{~nm})$ to the aluminum alloy is only $5 \%$ as shown in Fig. 1 (the relationship between absorption rate of aluminum A3003 and wavelength at a room temperature) [1][2]. Therefore, the processing efficiency is very low, and the high power laser system is required to obtain the sufficient deep penetration depth and the bead width. Though the high laser power can increase the penetration depth and bead width, the excessive heat input would lead to the deterioration of surface integrity and welding quality due to the spatter and the porosity [3].

On the other hand, the absorption rate of diode laser (hereafter LD) at the aluminum alloy shows a higher point at wavelength band of $800-850 \mathrm{~nm}$ as shown in Fig. 1 . The absorption rate of continuous LD at wavelength $\lambda=808 \mathrm{~nm}$ is about $15 \%$, which is 3 times higher than that of $\mathrm{Nd}: Y A G$ laser of $1064 \mathrm{~nm}$. Moreover, LD has increased its power and reduced its cost rapidly, since the semiconductor technology is remarkably developing day by day. Therefore, the welding technology of aluminum alloy by the combination of a pulsed Nd:YAG laser and a continuous LD was proposed, and the high efficiency and a high quality welding of aluminum alloy was reported [4].

However, the sufficient deep penetration depth could not be obtained at the beginning of laser scanning even with the superposition of LD. The higher peak power could make the penetration depth deeper, while it would lead to the risk of the deterioration of surface integrity. If the deep penetration depth could be obtained from the beginning of laser scanning with the better surface integrity, it is considered that this process is very useful for the industrial application.

From the viewpoints mentioned above, in this study, the pre-heating pulse method is proposed with the spatial superposition of a pulsed Nd:YAG laser and continuous LD in the welding of aluminum alloy to perform the deep penetration depth at the beginning of laser scanning. The effects of superposed LD on welding characteristics were investigated by using the experimental and the numerical analysis.

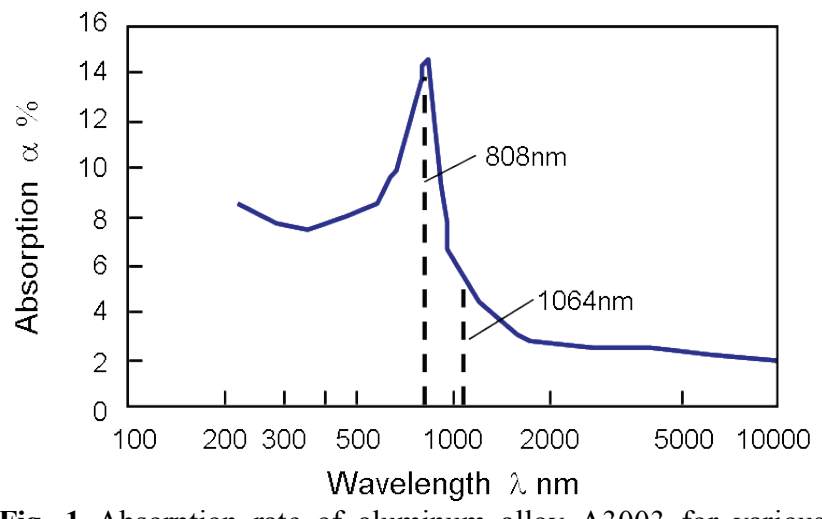

Fig. 1 Absorption rate of aluminum alloy A3003 for various wavelengths 


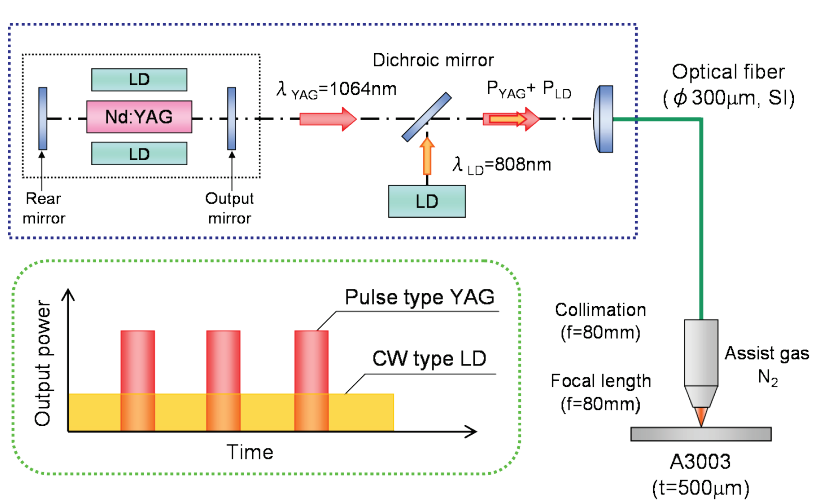

Fig. 2 Schematic diagram of laser irradiation system

Table 1 Specifications of pulsed Nd:YAG laser and continuous LD

\begin{tabular}{c|c|c}
\hline & Nd:YAG & LD \\
\hline Average power & $250 \mathrm{~W}$ & $65 \mathrm{~W}$ \\
\hline Max. peak power & $2.5 \mathrm{~kW}$ & - \\
\hline Wavelength & $1064 \mathrm{~nm}$ & $808 \mathrm{~nm}$ \\
\hline Pulse repetition rate & $1-500 \mathrm{~Hz}$ & $\mathrm{CW}$ \\
\hline Pulse width & $0.08-1.2 \mathrm{~ms}$ & $\mathrm{CW}$ \\
\hline
\end{tabular}

Table 2 Physical properties of aluminum A3003

\begin{tabular}{l|c}
\hline Specific heat & $900 \mathrm{~J} /(\mathrm{kg} \cdot \mathrm{K})$ \\
\hline Thermal conductivity & $237 \mathrm{~W} /(\mathrm{m} \cdot \mathrm{K})$ \\
\hline Density & $2.73 \mathrm{~g} / \mathrm{cm}^{2}$ \\
\hline Poisson's ratio & 0.33 \\
\hline Young's modulus & $70 \mathrm{kN} / \mathrm{mm}^{2}$ \\
\hline Coefficient of thermal expansion & $2.4 \times 10^{-6} / \mathrm{K}$ \\
\hline
\end{tabular}

\section{Experimental procedures}

Figure 2 shows the schematic diagram of laser irradiation system. Table 1 shows the specifications of a pulsed Nd:YAG laser and a continuous LD used in this study. A pulsed Nd:YAG laser of $1064 \mathrm{~nm}$ in wavelength and a continuous LD of $808 \mathrm{~nm}$ in wavelength were superposed on the same beam axis by a dichroic mirror, and the superposed laser beam of two wavelengths were delivered to the processing head through an optical fiber of $300 \mu \mathrm{m}$ diameter with SI type. These laser beams were collimated and focused by a lens of $80 \mathrm{~mm}$ in the focal length. The bead on plate welding experiment was carried out at the focusing point with $\mathrm{N}_{2}$ assist gas of $300 \mathrm{kPa}$. The aluminum alloy A3003 of $0.5 \mathrm{~mm}$ thickness was used as a specimen, and its physical properties are shown in Table 2.

Figure 3 shows the measurement points for the surface temperature of the specimen during the laser irradiation. Three thermocouples of K type were set at the distance of $1.0 \mathrm{~mm}$ from the laser scanning line. The point "a" was the parallel point to the start of laser scanning, and the point "b" and "c" were located at $10 \mathrm{~mm}$ and $20 \mathrm{~mm}$ from the point "a" in the parallel direction to the laser scanning line. The output signals of these thermocouples were recorded as the surface temperature of the specimen by using a digital recorder (NEC Avio Infrared Technologies Co., Ltd.

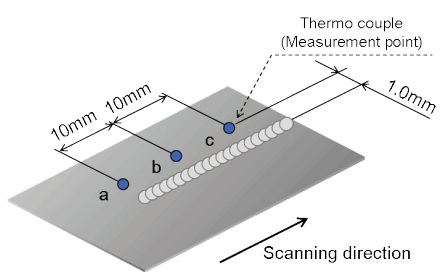

(a) Location of measuring points

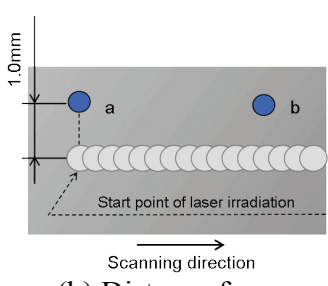
scanning line (b) Distance from

Fig. 3 Measurement points of surface temperature

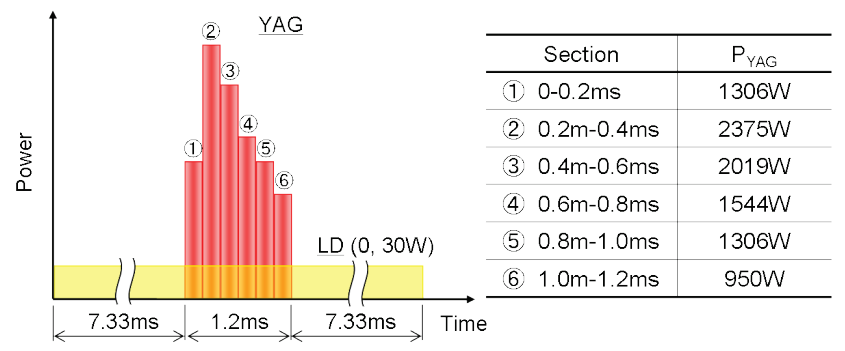

Fig. 4 Irradiation waveform of main Nd:YAG laser pulse

Omniace III RA2300).

Figure 4 shows the irradiation waveform of main $\mathrm{Nd}$ :YAG laser pulse, in which the power of $\mathrm{Nd}$ :YAG laser could be controlled every $0.2 \mathrm{~ms}$. In general, the sharp heating up and cooling down would lead to the welding defects such as blow holes and cracks. Therefore, the main pulse for the processing was controlled with a gradual increment and decrement of laser power during the pulse width $1.2 \mathrm{~ms}$. In the case of superposition of two laser beams, the irradiation of continuous LD started $7.33 \mathrm{~ms}$ before the main $\mathrm{Nd}$ :YAG pulse.

\section{Measurement and analysis of surface temperature}

A pulsed Nd:YAG laser and a continuous LD were focused into $300 \mu \mathrm{m}$ in diameter on the specimen surface under the following irradiation condition; the feed rate $F=30 \mathrm{~mm} / \mathrm{s}$, the pulse width of Nd:YAG laser $\tau=1.2 \mathrm{~ms}$, the pulse repetition rate of $\mathrm{Nd}$ :YAG laser $R p=120 \mathrm{~Hz}$, the peak power of pulsed Nd:YAG laser $P_{\text {pulse }}=2375 \mathrm{~W}$, and the average power of continuous LD $P_{L D}=30 \mathrm{~W}$. Figure 5 shows the measurement results of surface temperature without a continuous LD and with a continuous LD of $30 \mathrm{~W}$. The surface temperature of point "a" rose quickly from the beginning of laser irradiation for both cases. At the point " $b$ " and "c", the specimen surface was heated up by the preceding laser beam irradiation before the arrival of Nd:YAG laser pulse. After the Nd:YAG laser pulse passed the measurement point, the surface temperature decreased gradually. At every measurement point with a continuous LD of $30 \mathrm{~W}$, the surface temperature was approximately $50 \mathrm{~K}$ higher than that without a continuous LD. It was clear that the superposition of continuous LD made it possible to keep the specimen at higher temperature during the laser beam irradiation even during the interval time of $\mathrm{Nd}$ :YAG laser pulse.

Figure 6 shows the analytical model for the temperature distribution under the same condition of Fig. 5 (a) without a continuous LD. The general finite element program 'ANSYS Rev.11.0', in which the unsteady 


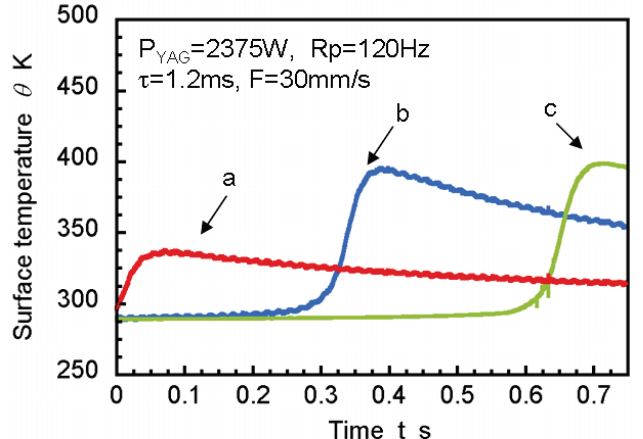

(a) YAG

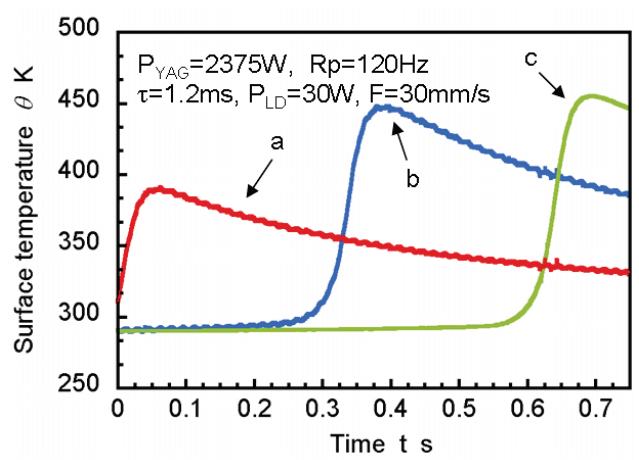

(b) $Y A G+L D$

Fig. 5 Measurement results of surface temperature without continuous LD and with continuous LD of $30 \mathrm{~W}$
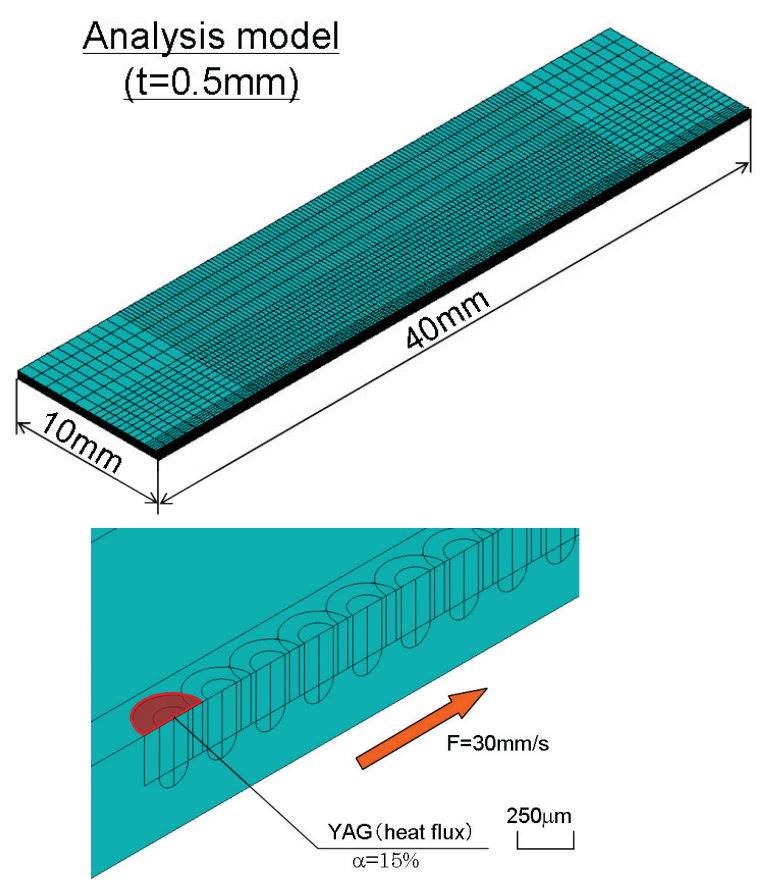

Fig. 6 Analytical model of temperature distributions

calculation is possible, was used for the numerical analysis. the main analytical conditions are shown in Table 3. A Nd:YAG laser irradiation was given as a heat flux from the specimen surface by the absorption rate $15 \%$, which was determined by the former investigation of penetration depth and bead width [5]. A pulse of Nd:YAG laser of $300 \mu \mathrm{m}$ spot diameter was irradiated at the pulse repetition rate $120 \mathrm{~Hz}$ and the scanning speed $30 \mathrm{~mm} / \mathrm{s}$. The
Table 3 Analytical conditions for the comparison of surface temperature without continuous LD

\begin{tabular}{l|c}
\hline Material & Pure aluminum $(\mathrm{t}=0.5 \mathrm{~mm})$ \\
\hline Beam spot diameter & $300 \mu \mathrm{m}$ \\
\hline Pulse repetition rate & $120 \mathrm{~Hz}$ \\
\hline Pulse width & $1.2 \mathrm{~ms}$ \\
\hline Feed rate & $30 \mathrm{~mm} / \mathrm{s}$ \\
\hline Coefficient of heat transfer & $35 \mathrm{~W} /\left(\mathrm{m}^{2} \cdot \mathrm{K}\right)$ \\
\hline Room temperature & $296 \mathrm{~K}$ \\
\hline
\end{tabular}

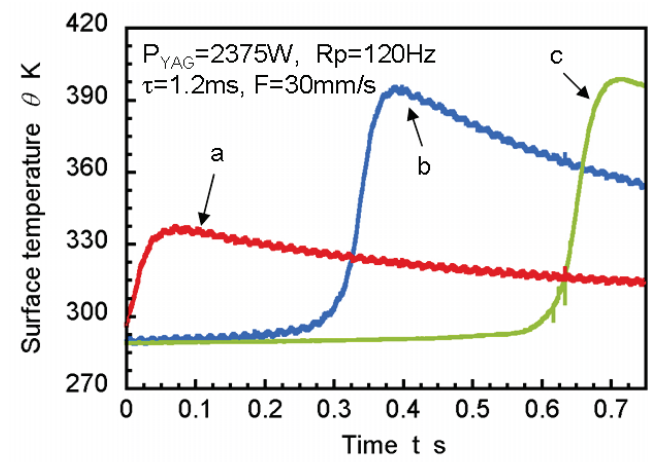

(a) Experiment

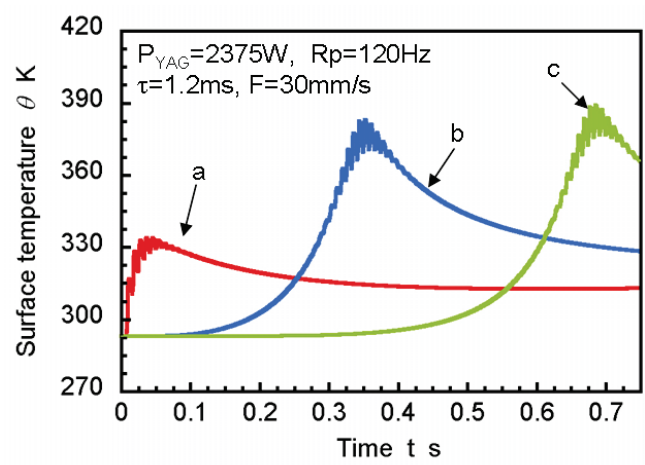

(b) Analysis

Fig. 7 Comparison of experimental and analytical results at the evaluation point without continuous LD

convective heat transfer condition of air was considered after the set time of laser irradiation. Except for the laser beam irradiated area, the convective heat transfer condition of air was also considered. The pure aluminum thermo physical properties of specimen were used for this analysis.

Figure 7 shows the experimental and the analytical results of surface temperature. The surface temperature of analysis corresponded to the experimental result, and it indicated that the experiment could measure the surface temperature of aluminum alloy during the laser irradiation. However, in the case of analysis, there was sharp fall down of temperature. It is considered that the values of thermal conductivity and specific heat of this analysis would be not suitable for A3003. This point would be investigated in the further study.

\section{Effect of pre-heating pulse method \\ 4.1 Experimental results}

Figure 8 shows irradiation waveform for the 


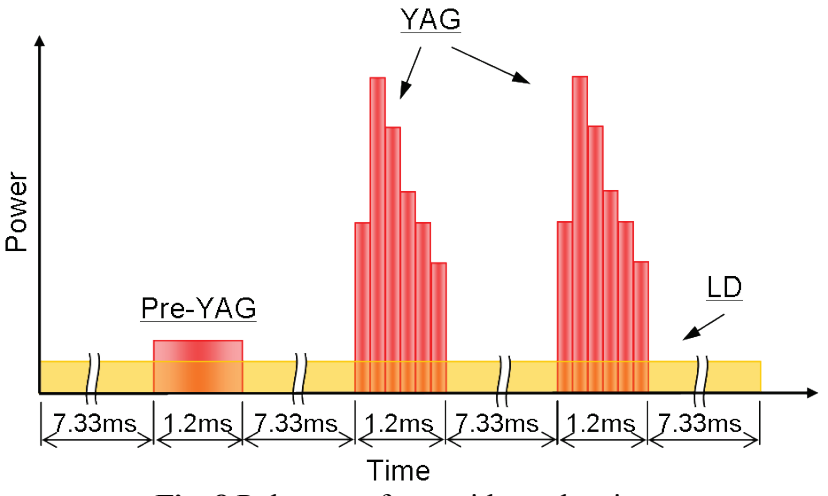

Fig. 8 Pulse waveform with pre-heating

Table 4 Experimental conditions for pre-heating pulse method

\begin{tabular}{l|c|c|c}
\hline & Pre-YAG & YAG & LD \\
\hline Peak power & $400 \mathrm{~W}$ & $2375 \mathrm{~W}$ & - \\
\hline Average power & - & - & $0,30 \mathrm{~W}$ \\
\hline Pulse width & $1.2 \mathrm{~ms}$ & $1.2 \mathrm{~ms}$ & $\mathrm{CW}$ \\
\hline Pulse repetition rate & - & $120 \mathrm{~Hz}$ & - \\
\hline Spot diameter & \multicolumn{3}{|c}{$300 \mu \mathrm{m}$} \\
\hline Feed rate & $30 \mathrm{~mm} / \mathrm{s}$ \\
\hline
\end{tabular}

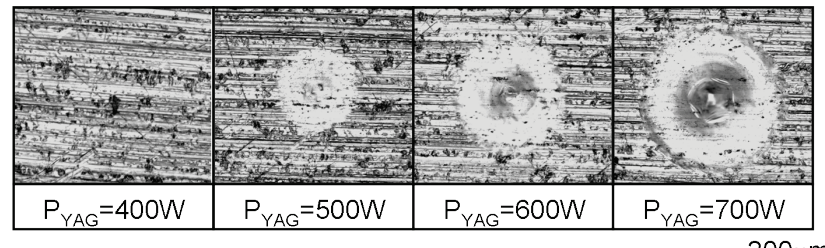

$\tau=1.2 \mathrm{~ms}$, Assist gas: $\mathrm{N}_{2}$

$200 \mu \mathrm{m}$

Fig. 9 Microphotographs of irradiated surface for various peak powers of $\mathrm{P}_{\mathrm{YAG}}$ by a single shot

pre-heating pulse method. Firstly, a continuous LD was irradiated on specimen surface. Secondly, the pre-heating pulse was added as a rectangular pulse waveform to increase the surface temperature at the beginning of laser scanning. After the pre-heating pulse, the main $\mathrm{Nd}$ :YAG laser pulses were irradiated. The experimental conditions for the pre-heating pulse method are shown in Table 4.

Figure 9 shows microphotographs of irradiated surface for various peak powers by the single laser shot. For more than peak power $500 \mathrm{~W}$, the specimen surface was melted and became glossy. It is considered that the glossiness of specimen surface might reflect a main Nd:YAG laser pulse. On the other hand, the specimen surface was not glossy in the case of peak power $400 \mathrm{~W}$, even the surface temperature rose high. Therefore, the peak power $400 \mathrm{~W}$ and the pulse width $1.2 \mathrm{~ms}$ of $300 \mu \mathrm{m}$ spot diameter was used for the pre-heating pulse method.

Figure 10 shows the experimental results of multi-shots at the beginning of laser scanning. Here, the power density of pulsed Nd:YAG laser was set in the transitional region between heat conduction welding and key-hole welding. For the normal case without the pre-heating pulse, the penetration depth became large gradually regardless of the superposition of a continuous LD (B, D). The penetration depth was not stable in the case

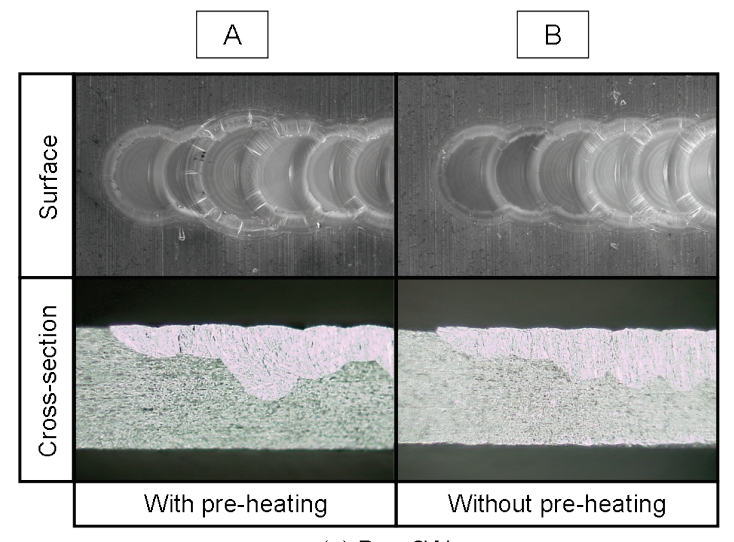

(a) $P_{\mathrm{LD}}=\mathrm{OW}$

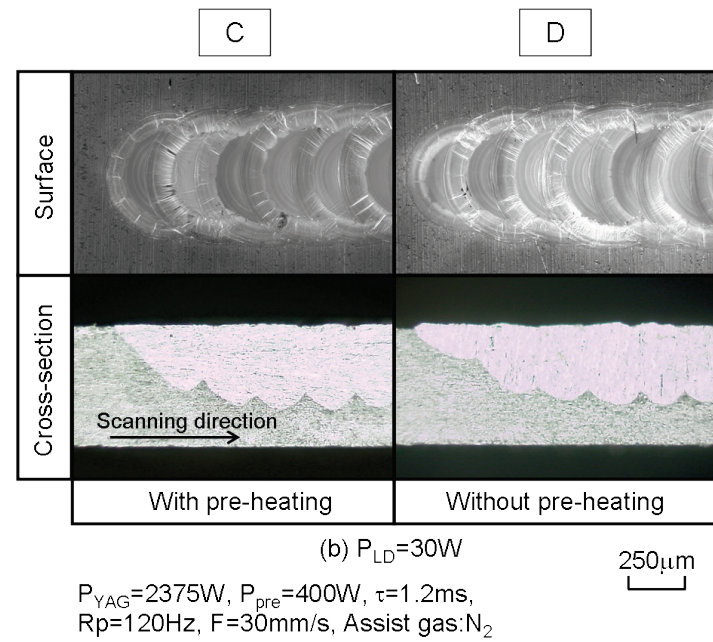

Fig. 10 Welding results at the beginning of laser scanning with and without pre-heating pulse

of pre-heating pulse method without the superposition of continuous LD (A). It is considered that the absorption rate of pulsed Nd:YAG laser was unstable at low specimen temperature, since the power density is the transition condition between heat conduction welding and key-hole welding. On the other hand, for the pre-heating pulse method with the superposition of continuous LD (C), the penetration depth could become large from the beginning of laser scanning by stable higher absorption of laser energy. Moreover, the stable welding phenomenon was obtained judging from the steady bead width and penetration depth. It indicated that both the pre-heating pulse and the superposition of continuous LD mutually led to the increase in melted volume at the beginning of laser scanning.

Figure 11 shows the bead width and the penetration depth at the beginning of laser scanning with and without the pre-heating pulse. At the first pulse, $100 \mu \mathrm{m}$ increase in the bead width and $200 \mu \mathrm{m}$ increase in the penetration depth were obtained by the combination of pre-heating pulse and continuous LD. Even if only the pre-heating pulse was applied at the beginning of laser scanning, the deeper penetration could not be obtained without the superposition of continuous LD. It is considered that the multiplication effect of pre-heating and continuous LD could lead to the effective welding state from the beginning of laser scanning. 


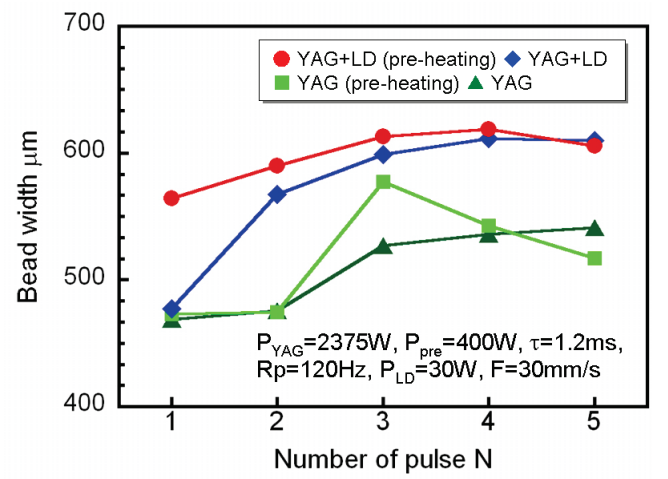

(a) Bead width

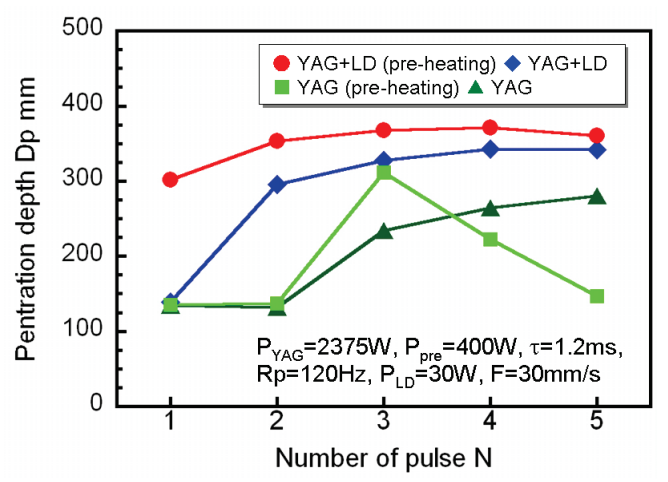

(b) Penetration depth

Fig. 11 Change of bead width and penetration depth for number of pulse

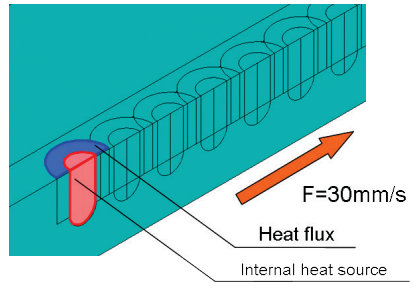

(a) With continuous LD

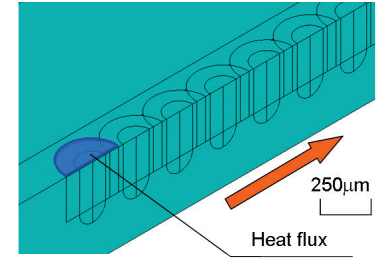

(b) Without continuous LD
Fig. 12 Analytical process for pre-heating pulse method with and without continuous LD

\subsection{Numerical analysis}

The temperature distributions were investigated by the numerical calculation in order to discuss the difference of welding phenomenon with and without the pre-heating pulse. The same analysis model shown in Fig. 6 was used for these analyses, and the analysis process at the beginning of laser scanning is shown in Fig. 12. In the case of superposition of continuous LD, the key-hole effect was assumed. Internal heat generation by the heating element of shape mixed column and hemisphere was considered as a heat source as shown in Fig. 12 (a). The total power of a pulsed Nd:YAG laser and a continuous LD was irradiated as an internal heat generation. A continuous LD was irradiated on the specimen surface except for pulsed $\mathrm{Nd}$ :YAG laser shot. The absorption rate of pulsed Nd:YAG laser was defined as $15 \%$ for a heat flux and $30 \%$ for an internal heat generation [5]. The pulse waveform was the same as shown Fig. 4. 30W continuous LD was given by the absorption rate as shown in Fig. 13 [5]. The

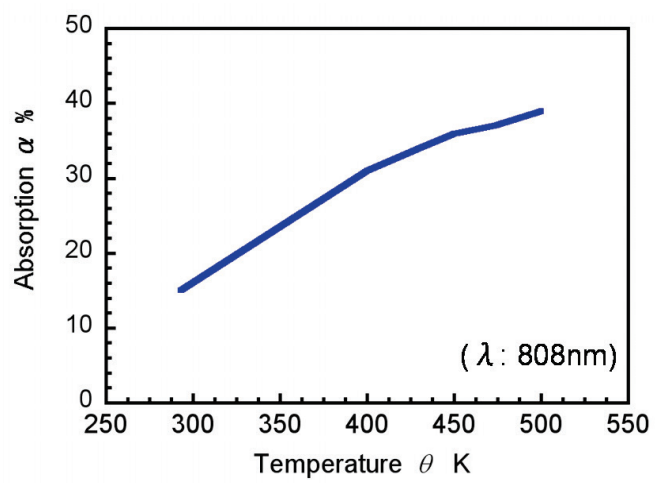

Fig. 13 Absorption rate of LD to aluminum alloy at $808 \mathrm{~nm}$

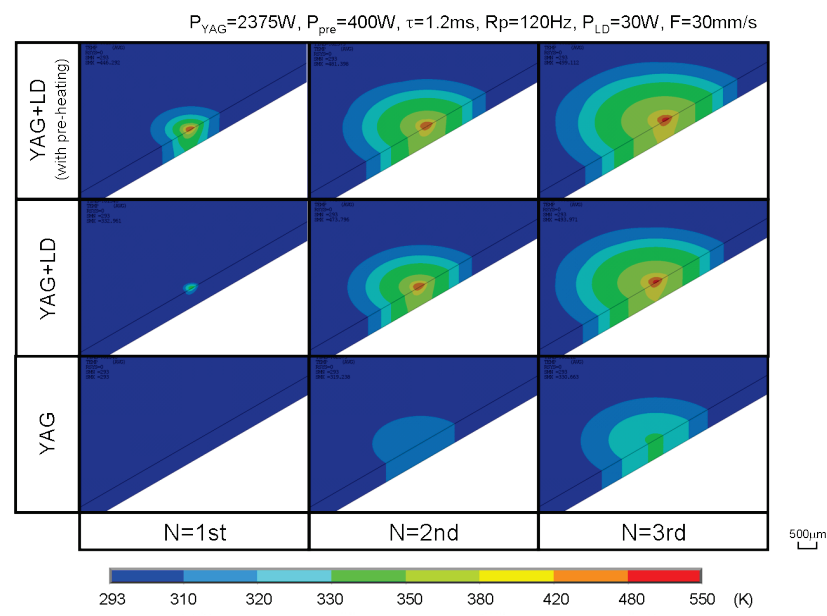

Fig. 14 Temperature distributions before irradiation of pulsed YAG laser

extrapolated value was used as the absorption rate of continuous LD for temperature region. Without the superposition of continuous LD, a pulsed Nd:YAG laser was given as a heat flux of absorption rate $15 \%$ as shown in Fig. 12 (b). The other analytical conditions were the same as Table 3 .

Figure 14 shows the analytical results of surface temperature distributions before the irradiation of pulsed YAG laser. In the case of only YAG laser, the surface temperature was the same as a room temperature before the first main YAG pulse, since there was no energy input. The surface temperature of YAG+LD increased slightly before the first main YAG pulse. On the other hand, the irradiation method of YAG+LD with the pre-heating pulse kept the specimen surface at the higher temperature even before the first main YAG pulse. Therefore, it is considered that the pre-heating pulse would increase the surface temperature, and a continuous LD would keep the high temperature.

Figure 15 shows the surface temperature of spot center before the irradiation of main YAG laser pulse. By using both pre-heating pulse and continuous LD, the first surface temperature rose approximately $200 \mathrm{~K}$ higher than that of YAG+LD without pre-heating pulse. The absorption rate of $\mathrm{Nd}$ :YAG laser to aluminum alloy increases drastically more than $900 \mathrm{~K}$, melting point of aluminum alloy. In the case without pre-heating pulse and continuous LD, a pulsed Nd:YAG laser would be irradiated on specimen surface at the low temperature firstly, which would lead 


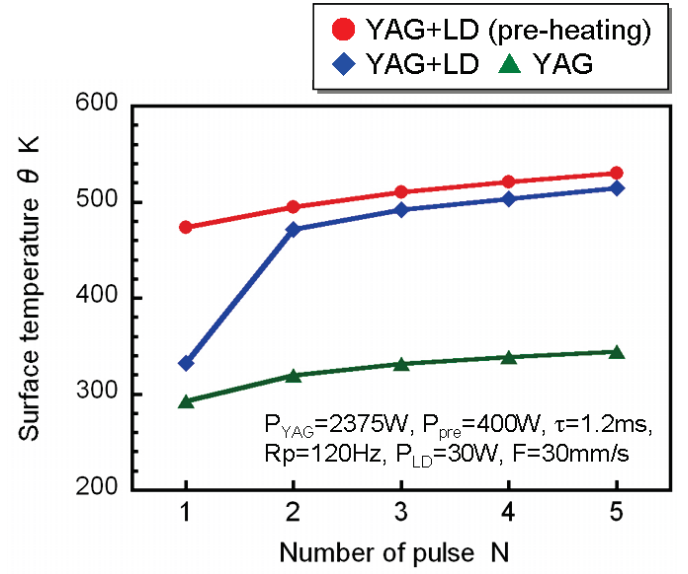

Fig. 15 Surface temperatures of spot center before irradiation of main $\mathrm{Nd}$ :YAG laser pulse

to the unstable absorption of a pulsed Nd:YAG laser beam. On the other hand, it is easy to reach the melting point in the case with pre-heating pulse and continuous LD compared with the case of only Nd:YAG laser irradiation. Therefore, it was considered that the energy of pulsed $\mathrm{Nd}$ :YAG laser was absorbed effectively and stably to the specimen surface because of its higher surface temperature even from the beginning of laser scanning with pre-heating pulse and continuous LD.

\section{Conclusions}

In this study, the pre-heating pulse method was proposed with the spatial superposition of a pulsed Nd:YAG laser and continuous LD in the welding of aluminum alloy, and its characteristics were investigated. Main conclusions obtained in this study are as follows.

(1) During the interval time of laser irradiation, the surface temperature of specimen was kept higher by the superposition of continuous LD.

(2) A pulsed Nd:YAG laser was absorbed effectively from the beginning of laser scanning by the pre-heating pulse method with the superposition of continuous LD due to the high surface temperature of specimen.

(3) The combination of pre-heating pulse and continuous LD made the welding phenomenon stable from the beginning of laser scanning by stable absorption of pulsed Nd:YAG laser.

\section{References}

[1] National Astronomical Observatory Institute.: Chronological Scientific Tables 2001, (2001) p. 523.

[2] N. Abe N: Preface to Topical Papers on Direct Applications of High-Power Semiconductor Lasers, The review of Laser Engineering, vol.31, No. 5, (2003) p. 323.

[3] T. Sakurai, Y. Nakagawa: Development of LD+YAG Hybrid Laser, Proceedings of the 62nd Laser Materials Processing Conference (2004) pp. 102-110.

[4] T. Sakagawa, Y. Okamoto, Y. Uno, S.Haraguchi and S. Nakashiba: High-efficiency Welding of Aluminum Alloy by Hybrid System Combined pulsed Nd:YAG Laser and Diode Laser, Proceedings of 26th International Congress on Applications of Lasers \& Electro-Optics 2009, (2009) pp.1008-1014.

[5] S. Haraguchi, Y. Okamoto, Y. Uno, T. Sakagawa and S. Nakashiba: Investigation on Welding Phenomenon for Aluminum Alloy by Superposition of Pulsed YAG Laser and Diode Laser, Proceedings of International Conference on Leading Edge Manufacturing in 21st Century (in Osaka), (2009), pp.573-576.

(Received: June 07, 2010, Accepted: October 13, 2011) 